\title{
Predicting maximum voluntary ventilation in normal healthy individuals using indirect inspiratory muscle strength measurements: a correlation study
}

\author{
Rohit Sontakke $^{1}$, Mangesh Deore ${ }^{2}$, Dhara Kothari ${ }^{3}$ \\ ${ }^{1}$ Masterskill University College of Health, Cheras, Malaysia; rohitrohits@yahoo.com \\ ${ }^{2}$ Sancheti Institute of Orthopedics, Pune, India \\ ${ }^{3}$ Nivara Physiotherapy Center, Pune, India
}

Received 17 November 2009; revised 7 December 2009; accepted 15 December 2009.

\begin{abstract}
Maximum Voluntary Ventilation (MVV), one of the components of Pulmonary Function Testing (PFT), has multiple uses. Various factors including the inspiratory muscle strength (IMS) influence its magnitude. Our aim was to quantify the IMS indirectly using an economical and non invasive bedside assessment tool, determine its association with MVV and then develop a predictive equation for MVV. 41 healthy non-athletic physical therapy students participated in the study. IMS measurement was performed with a sphygmomanometer. Average of the three net deflections in sphygmomanometer following deepest possible breaths was taken as indirect measurement of IMS in $\mathrm{mm}$ of $\mathrm{Hg}$. MVV was measured according to ATS guidelines using a spirometer. Results from the data analysis revealed a significant correlation between IMS and $\operatorname{MVV}(r=0.83, p<0.001)$ and the coefficient of determination $=0.68$. So, we developed a regression equation: $Y=1.9669(X)+49.838$ with SEE: $13.02 \mathrm{~L} / \mathrm{min}$ and ANOVA for the equation was $(F=68.9, p<0.001)$. Hence, it can be concluded that a strong correlation between the indirect IMS and MVV was established and a predictive equation to estimate MVV was developed. This equation proved to have a high predictive value with a small error of estimation. This indicates that the value of the indirect IMS measurement obtained using the sphygmomanometer can be used to estimate MVV in normal healthy individuals without the use of a conventional spirometer.
\end{abstract}

Keywords: Healthy Physical Therapy Students; Maximum Voluntary Ventilation; Inspiratory Muscle Strength; Regression Equation; Sphygmomanometer

\section{INTRODUCTION}

Evidence-based support for pulmonary rehabilitation in the management of patients with chronic respiratory dysfunction has grown tremendously, and this comprehensive intervention has clearly demonstrated to reduce dyspnea and health care costs, increase exercise performance, and improve health-related quality of life (HRQL) [1-6].

One of the factors responsible for exercise limitations and reduced HRQL in patients with respiratory disorders is dyspnea $[7,8]$. Weakness or mechanical inefficiency of the respiratory muscles results in a mismatch between central respiratory motor output and achieved ventilation. This mismatch can also explain the dyspnea experienced by patients with neuromuscular diseases affecting the respiratory musculature [9] and in patients with respiratory muscle fatigue [10]. As the pressure-generating capacity of the respiratory muscles falls and as the ratio of the pressures produced by the respiratory muscles to the maximum pressure that can be achieved increases dyspnea progressively worsens [11]. Since there is an established association between respiratory muscle dysfunction and dyspnea, an improvement in respiratory muscle function with inspiratory muscle training (IMT) could lead to a reduction in dyspnea [12,13]. A meta-analysis of IMT in 17 clinical trials found limited support for its use in terms of improving pulmonary function, respiratory muscle strength and endurance, exercise capacity, and functional status in patients with COPD [14-16]. A consistent improvement in baseline dyspnea index (BDI) and transitional dyspnea index was shown following IMT [17]. Also, fewer dyspnea was reported by patients who used IMT with a threshold loading device at $30 \%$ of Pimax nevertheless, those who used either a very light load or sham treatments also reported less dyspnea [18]. Inspiratory muscle training has also been found to reduce dyspnea during exertion not only in patients with respiratory ailments but also in normal healthy individu- 
als [19].

There are many individuals with chronic cardiopulmonary disorders for which the underlying pathophysiology cannot be corrected and this, in turn, frequently results in long-term disability for the patient [20]. A pulmonary rehabilitation programme is essential for these patients. For a successful rehabilitation program, accurate assessment is very important. There are variety of measures to assess the functioning of respiratory system which include tests of flows and volumes [21], tests of respiratory muscle strength [22], endurance [23], fatigue [24], and chest wall function analysis [25]. Routine measurements of respiratory function (i.e., volumes, flows and indices of gas exchange) are non specific in relation to diagnosis but give useful indirect information about respiratory muscle performance. More frequently, these measurements are of use in assessing the severity, functional consequences and progress of patients with recognized respiratory muscle weakness [21]. Amongst the various tests for flows and volumes, maximum voluntary ventilation (MVV) is a parameter that reflects lung volume changes, respiratory muscle functioning, compliance of the thorax lung complex and airway resistance [26]. MVV is defined as the maximum amount of air that a subject can breathe over a specified period of time (12 seconds for normal subjects) and is expressed in L/min [27]. It can be used as a tool for assessment of respiratory muscle weakness [21]. The accurate estimation of MVV is critical for interpretation of maximal sustainable ventilation (MSV). MSV is a measure of endurance of ventilatory muscles and is expressed as a fraction of MVV [23].

Direct measurements of respiratory muscle strength are conducted using invasive as well as noninvasive techniques. Both types of techniques require sophisticated instrumentations. Measurement of Pi max (Peak Inspiratory Pressure) is one of the most commonly used techniques for quantification of IMS. Measurement of Pi max, though is a non-invasive method, the device to measure Pi Max (Manovacuometer) is not routinely available in Indian Physiotherapy set ups as it is not cost effective. Accurate estimation of MVV value according to ATS/ERS guidelines [27] requires a sophisticated machine and the maneuver itself requires coordination, motivation, understanding and may induce fatigue, giddiness and bronchospasm in the candidate who undergoes the test. Also, this maneuver is not recommended in the individuals either having or suspected respiratory muscle weakness [21]. In order to overcome the aforementioned limitations of various measurement techniques, we measured the IMS using a sphygmanometer. This device is easily available in any physical therapy set-up, and is inexpensive. The purpose of our study was to explore the relationship between the values of IMS obtained using the sphygmanometer and MVV measured using the spirometer. If a strong relationship exists, then a regression equation to estimate MVV from the indirectly measured IMS values can be obtained. This equation will serve the purpose of guiding clinical decision making without the need of a sophisticated instrumentation and/or causing discomfort to our subjects.

\section{MATERIALS AND METHODS}

\subsection{Ethical Approval}

Institutional ethical committee approval was obtained from Sancheti Institute for orthopedics and rehabilitation. All the subjects were given a thorough explanation of the procedure and a written informed consent was obtained before participating in the study.

\subsection{Participants}

The study population consisted of normal healthy physiotherapy students from Sancheti Institute College of Physiotherapy, Pune. All the subjects were non- athletes, non smokers and healthy. A total number of 41 subjects participated in the study. Demographics are presented in Table 1.

\subsection{Methods}

A gap of at least four hours was given between the food intake and actual procedure to minimize the hindrance in the diaphragm excursion. The subjects were also instructed to empty the bladder before the procedure.

\subsection{Estimation of Indirect Inspiratory Muscle Strength (IMS)}

The equipments used for this measurement were a pediatric size blood pressure cuff, a leather belt and an aneroid sphygmomanometer. The subject was positioned supine on a plinth with hip knee flexion. Velcro straps of the blood pressure cuff were removed and it was secured to the candidates chest two $\mathrm{cm}$ below the xiphoid process. The leather belt was used for this purpose. The blood pressure cuff was inflated to a baseline pressure of $20 \mathrm{mmHg}$ which was maintained. The candidate was then instructed to take the deepest possible breath starting from functional residual capacity (FRC) and hold it for 1 second until we noted the net deflection in manometer. The cuff was deflated and the entire procedure was repeated two more times with a rest of ten minutes in between each measurements. The average of the three

Table 1. Demographic data.

\begin{tabular}{cc}
\hline Variable & Mean $( \pm$ SD) \\
\hline Age $(\mathrm{yrs})$ & $21( \pm 3)$ \\
Males(n) & 16 \\
Females(n) & 25 \\
IMS (mmHg) & $49( \pm 9)$ \\
MVV (L/min) & $110( \pm 21)$ \\
\hline
\end{tabular}


net deflections was taken as an indirect measurement of inspiratory muscle strength, expressed in $\mathrm{mm} \mathrm{Hg}$.

\subsection{Estimation of Maximum Voluntary Ventilation}

A device RMS-Respirator ${ }^{\circledR}$ was used for measuring MVV. The device consisted of a flow sensor and a central port attached to a desk top computer. The device was calibrated using the standard procedure before the commencement of the study. The calibration was ensured periodically during the data collection. After the IMS was measured, the candidate was given a rest of $30 \mathrm{~min}$. The candidate underwent the slow vital capacity (SVC) maneuver and the ratio of total inspiratory time to total respiratory cycle time (Ti/Ttot) ratio was measured. The $\mathrm{Ti} / \mathrm{Tt}$ tot ratio between 0.35 and 0.4 ruled out the inspiratory muscle fatigue. The candidate was given a demonstration of the MVV maneuver to make sure that he/she had understood the procedure. The MVV was measured for 12 seconds using the ATS/ ERS standard procedure [27].

\subsection{Data Analysis}

Microsoft Excel analysis tool pack was used for analyzing the data. Pearson correlation coefficient was calculated to study the strength of the association between indirect IMS and MVV. Regression equation was developed by manual calculation. In order to evaluate the accuracy of prediction, coefficient of determination and standard error of estimation (SEE) were calculated. Analysis of variance for regression equation (ANOVA) was performed. Level of significance was set at 0.05 . Manual calculation of the statistical tests was performed as per the available guidelines [28].

\section{RESULTS}

\subsection{Regression Equation}

The Pearson correlation coefficient (r) obtained was 0.83 $(\mathrm{p}<0.001)$. This showed a strong correlation exists between MVV and indirect IMS. This has been depicted in Figure 1. As the strong correlation was established, we developed a regression equation. It was as follows. $\mathbf{Y}=$ 1.9669(X) + 49.838. In this equation $Y$ denotes the MVV and X denotes the indirect IMS.

\subsection{Analysis of Residuals}

Residual is the difference between the actual MVV value as obtained using the spirometer and the predicted MVV value as obtained from the regression equation. Actual MVV values plotted against the residuals (the difference between actual and predicted MVV values) demonstrated that they were evenly and randomly distributed

®RECORDER'S MEDICARE SYSTEM'S RMS MEDSPIROR. Platform Win 98.Version1.0

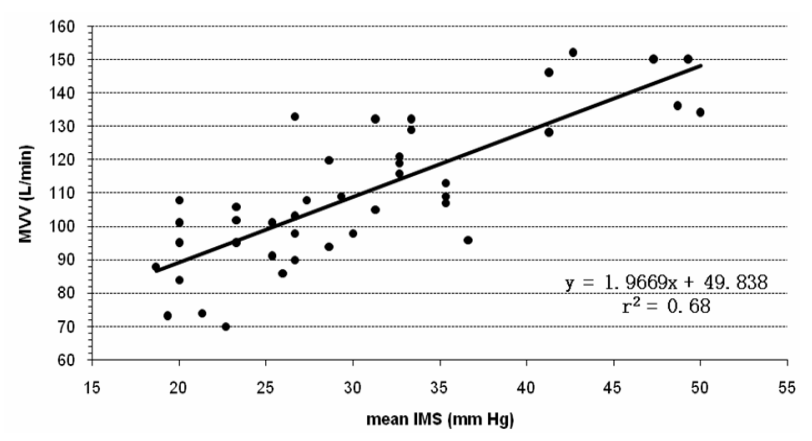

Figure 1. Regression equation and $X Y$ scatter showing the association between indirect IMS and MVV.

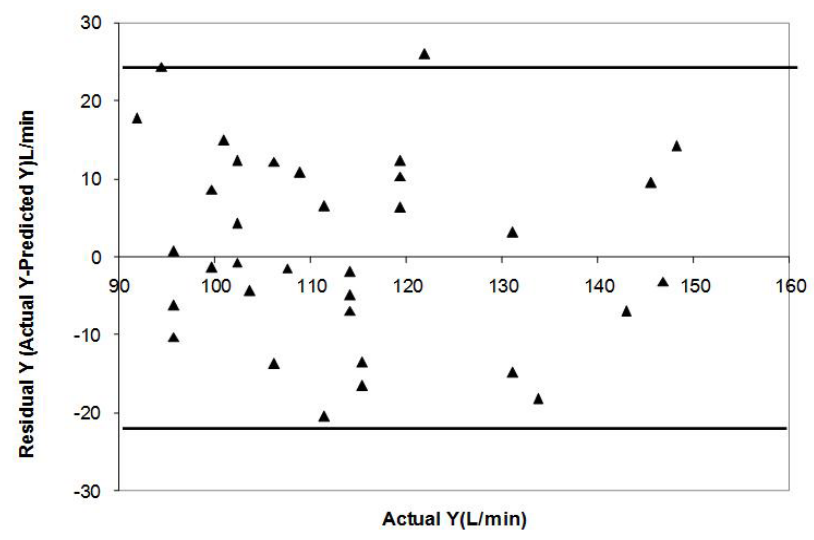

Figure 2. Analysis of residuals: Actual Y Vs residual Y value. As the actual value of the MVV increases, the error associated with the predicted MVV value does not increase.

about the regression line confirming the assumptions of the regression equation obtained as shown in Figure 2. As depicted in Figure 2, the residuals obtained in our study were evenly and randomly distributed about the regression line. This confirms that the assumptions of regression equation were met.

\subsection{Accuracy of Prediction}

The coefficient of determination r2 was 0.68 . This indicates that $68 \%$ change in the $\mathrm{Y}$ variable is been explained by $\mathrm{X}$ variable. Standard error of estimation (SEE) for the regression equation was $13.02 \mathrm{~L} / \mathrm{min}$. If we take into account the normal values of the MVV in healthy individuals, this is a very negligible value suggesting that our equation has a good accuracy of prediction. ANOVA (analysis of variance) was done for the regression equation which came to be significant (Calculated $F=68.9$, critical value of $(0.05) F(1,39)=4.08)$. This denotes that the obtained correlation between $\mathrm{X}$ and $\mathrm{Y}$ is not due to a chance.

\section{DISCUSSION}

The results of the study demonstrate that MVV is 
strongly correlated with the indirect IMS and MVV can be predicted with a good accuracy using the indirect IMS in a group of normal healthy college students. In addition, the prediction equation has proved to have excellent validity when ANOVA and analysis of the residuals was done. Coefficient of determination of 0.68 suggests that $68 \%$ of the variance in MVV can be explained by variance in IMS. This is because in addition to IMS, expiratory muscle strength, compliance of lungs and chest wall, airway resistance also contributes to MVV [26].

Peak inspiratory pressure (Pi Max) is one of the simplest and commonly used tool that is used for quantification of IMS. But, this device is not routinely available in physical therapy set ups in India. Due to this reason we chose to quantify the IMS indirectly instead of using Pi max.

The reasons for trying to quantify the indirect IMS and then predicting MVV from it are multiple. Patients could possibly save time, expenses, and invasive procedures if an accurate prediction of their maximum voluntary ventilation could be made from their indirect IMS. A common equation used for MVV estimation is MVV = $35.0 \times$ FEV1. The usefulness of this equation has been established in predicting MVV, in American African girls [29]. This method may underestimate the maximum exercise ventilation in COPD patients [31] Also, this equation requires the actual value of FEV1 to be substituted which can be obtained only with the use of a spirometer. In contrast to, these limitations, our study enables the clinicians to estimate the MVV using the regression equation which requires IMS value which can be obtained without the need of a spirometer or any other complicated device.

Applicability of Caucasian regression equations was also studied on Indian population for prediction of forced vital capacity (FVC), forced expiratory volume in first second (FEV 1) [31]. The conclusion of this study was that the commonly used Caucasian prediction equations, or a fixed percentage of their predicted values, were leading to the improper interpretation of the data obtained; that is, there was a significant difference between the values obtained using the equation and the values obtained by actual performing the maneuver. This happened in a significant proportion of patients and the study reflected that there is a need to assess performance of more than one regression equation before choosing any single prediction equation in Indian population as the most important step in diagnosing abnormality of lung function in individuals is to define whether they are within or out side the healthy subjects range. Though in this study it was the vital capacity (VC) which was studied, we can still expect that we can apply the conclusions from this study to the MVV also as VC is been found to have proportionate reductions to MVV [21].

None of these studies which studied the predictive equations for MVV had focused on the direct or indirect IMS measurement. Also the studies which focus on IMS have used the devices which are still expensive in India and not routinely available in Indian Physiotherapy set ups. Our technique of estimating IMS was inexpensive, non invasive, uncomplicated and can be used in any small clinical set up and also in ambulatory care set up. Thus, our study overcomes the previously mentioned drawbacks of the techniques for quantification of IMS and MVV in Indian set up. Here, it was the chest expansion that is, overall chest wall movement that was used for assessment of IMS. Throughout the study it was taken into account that the changes in the intrathoracic blood volume could have resulted in the difference between actual lung volume change and change in the volume of the thorax or simply the chest expansion. But this difference being negligible has not influenced our results [25]. This means that the chest wall motion has reflected the volume changes occurring in the lungs during breathing as there in no disparity between volume changes in the lungs and volume changes of the thoracic wall. One more confounding factor can be the elasticity of the chest wall tissues. But, it has been shown that the tissues of the chest wall being essentially incompressible, volume changes of the chest wall surface are nearly equal to volume changes of the lungs and can be used for indirect measure of IMS [25]. Regression equation will be useful only when the IMS value lies between $18.67 \mathrm{~mm} \mathrm{Hg}$ to $50 \mathrm{~mm} \mathrm{Hg}$ and for the age group of 18 to 24 yrs. The quantification indirect inspiratory muscle strength needs to be studied in wider age range and diseased population to increase its clinical applicability. Also, the contribution of expiratory muscle strength to MVV was not taken into account. Along with this, reliability and validly of this new tool for evaluating IMS needs to be studied.

\section{CONCLUSIONS}

A strong correlation between the indirect IMS and MVV was established. A regression equation was developed. This equation was tested for its ability to accurately predict the MVV and it proved to have a high predictive value with a small error of estimation. This signifies that by substituting the value of the indirect IMS in the obtained equation, we can estimate MVV in normal healthy individuals. This prediction will have a good accuracy.

\section{REFERENCES}

[1] Von Leupoldt, A., Hahn, E., Taube, K., Schubert-Heukeshoven, S., Magnussen and H., Dahme, B. (2008) Effects of 3-week outpatient pulmonary rehabilitation on exercise capacity, dyspnea, and quality of life in COPD. 
Lung, 186(6), 387-391.

[2] Güell, R., Resqueti, V., Sangenis, M., Morante, F., Martorell, B., Casan, P., et al. (2006) Impact of pulmonary rehabilitation on psychosocial morbidity in patients with severe COPD. Chest, 129(4), 899-904.

[3] Verrill, D., Barton, C., Beasley, W. and Lippard, W.M. (2005) The effects of short-term and long-term pulmonary rehabilitation on functional capacity, perceived dyspnea, and quality of life. Chest, 128(2), 673-683.

[4] Cecins, N., Geelhoed, E. and Jenkins, S.C. (2008) Reduction in hospitalization following pulmonary rehabilitation in patients with COPD. Australian Health Review, 32(3), 415-422.

[5] Kyung, K.A. and Chin, P.A. (2008) The effect of a pulmonary rehabilitation programme on older patients with chronic pulmonary disease. Journal of Clinical Nursing, 17(1), 118-125.

[6] Hui, K.P., Alison, B. and Hewitt, A.B. (2003) Simple pulmonary rehabilitation program improves health outcomes and reduces hospital Utilization in patients with COPD. Chest, 124, 94-97.

[7] Nici, L., Donner, C., Wouters, E., Zuwallack, R., Ambrosino, N., Bourbeau, J., et al. (2006) American thoracic society/European respiratory society statement on pulmonary rehabilitation. American Journal of Respiratory and Critical Care Medicine, 173, 390-1413.

[8] Hill, K., Jenkins, S.C., Hillman, D.R. and Eastwood, P.R. (2004) Dyspnea in COPD: Can inspiratory muscle training help? Australian Journal of Physiotherapy, 50(3), 169-180.

[9] Rochester, D. (1993) Respiratory muscles and ventilatory failure. American Journal of Medical Science, 305, 394402.

[10] Altose, M.D. (1992) Respiratory muscles and dyspnea. Seminars in Respiratory Medicine, 13, 1-6.

[11] Killian, K.J. and Jones, N.L. (1988) Respiratory muscles and dyspnea. Clinics in Chest Medicine, 9, 237-248.

[12] Weiner, P., Magadle, R., Beckerman, M., Weiner, M. and Berar-Yanay, N. (2004) Maintenance of inspiratory muscle training in COPD patients: One year follow-up. European Respiratory Journal, 23(1), 61-65.

[13] Battaglia, E., Fulgenzi, A., Bernucci, S., Giardini, M.E. and Fererro, M.E. (2006) Home respiratory muscle training in patients with chronic obstructive pulmonary disease. Respirology. 11(6), 799-804.

[14] Smith, L., Cook, D., Guyatt, G., Madhavan, J. and Oxman, A. (1992) Respiratory muscle training in chronic airflow limitation: 8 meta-analyses. American Review of Respiratory Disease, 145, 533-539.

[15] Padula, C.A. and Yeaw, E. (2006) Inspiratory muscle training: Integrative review. Research and Theory for Nursing Practice, 20(4), 291-304.

[16] Lötters, F., van Tol, B., Kwakkel, G. and Gosselink, R. (2002) Effects of controlled inspiratory muscle training in patients with COPD: A meta-analysis. European Respiratory Journal, 20(3), 570-576.

[17] Harver, A., Mahler, D.A. and Daubenspeck, J.A. (1989) Targeted inspiratory muscle training improves respiratory muscle function and reduces dyspnea in chronic obstructive pulmonary disease. Annals of Internal Medicine, 111, 117-124.

[18] Kim, M., Larson, J., Covey, M., Vitalo, C., Alex, C. and Patel, M. (1993) Inspiratory muscle training in patients with chronic obstructive pulmonary disease. Nursing Research, 42, 356-362.

[19] McConnell, A.K. and Romer, L.M. (2004) Dyspnoea in health and obstructive pulmonary disease: The role of respiratory muscle function and training. Sports Medicine, 34(2), 117-132.

[20] American Thoracic Society. (1999) Dyspnea: Assessment, mechanism and management: A consensus statement. American Journal of Respiratory and Critical Care Medicine, 159, 321-340.

[21] Gibson, G.J., Whitelaw, W. and Nikolaos, S.N. (2002) Tests of over all respiratory function. ATS/ERS statement on respiratory muscle testing. American Journal of Respiratory and Critical Care Medicine, 166, 521-526.

[22] Green, M., Road, J. and Sieck, G.C. (2002) ATS/ERS statement on respiratory muscle testing. American Journal of Respiratory and Critical Care Medicine, 166, 528-542.

[23] Clanton, T., Calverly, P.M. and Celli, B.R. (2002) Tests of respiratory muscle endurance. ATS/ERS statement on respiratory muscle testing. American Journal of Respiratory and Critical Care Medicine, 166, 559-569.

[24] Supinski, G.S., Fitting, J.W. and Bellemare, F. (2002) Assessment of Respiratory Muscle fatigue. ATS/ERS statement on respiratory muscle testing. American Journal of Respiratory and Critical Care Medicine, 166, 571-578.

[25] Loring, S.H., Troyer, A. and Grassino, A,E. (2002) Assessment of Chest Wall Function. ATS/ERS statement on respiratory muscle testing. American Journal of Respiratory and Critical Care Medicine, 166, 580-586.

[26] Sheldon, R.L. (2000) Pulmonary Function Testing. In Wilkins, R.L., Krider, Susan, J., Clinical assessment in respiratory care. 4th Edition, Mosby, St. Louis, 141-160.

[27] Millre, M.R., Hankinson, J. and Brusasco, V. (2005) Maximum voluntary ventilation. Standardisation of spirometry: SERIES “ATS/ERS task force: Standardization of lung function testing”. European Respiratory Journal, 26, 331-334.

[28] Portney, L.G. and Watkins, M.P. (2009) Foundations of clinical research applications to practice. 3rd Editon, Prentice Hall, New Jersey, 539-567.

[29] Fulton, J.E., Pivarnik, J.M., Taylor, W.C., Snider, S.A., Tate, A.L. and Frankowski, R.F. (1995) Prediction of maximum voluntary ventilation (MVV) in African-American adolescent girls. Pediatric Pulmonology, 20(4), 225233.

[30] Pride, N.B. (2001) Tests of forced inspiration and expiration. Clinics in Chest Medicine, 22(4), 599-622.

[31] Aggarwal, A.N., Gupta, D., Behera, D. and Jindal, S.K. (2005) Applicability of commonly used Caucasian prediction equations for spirometry interpretation in India. Indian Journal of Medical Research, 122(2), 153-164. 